\title{
L'anesthésiste, le troisième homme
}

\section{The anaesthetist, the third man}

\author{
E. Morau \\ C Springer-Verlag France 2011
}

L'anesthésie-réanimation est une spécialité jeune qui, au fur et à mesure de son développement, a vu se créer des sousspécialités liées à des profils de patients particuliers comme la pédiatrie ou orientées sur un type de chirurgie (orthopédie, viscérale, etc.). L'anesthésie-réanimation obstétricale est une de celles-là, orientée vers les femmes enceintes. Au cours des 20 dernières années, en raison de l'essor de l'analgésie péridurale, l'implication des anesthésistes en secteur obstétrical a été croissante avec en 2011 de plus en plus d'anesthésistes travaillant exclusivement en secteur obstétrical. Cela s'est traduit par une organisation propre avec création de postes d'infirmier anesthésiste dédiés à l'obstétrique et de liste de garde d'interne de spécialité.

Le récent rapport sur la situation périnatale en France en 2010 [1] nous apporte des éléments chiffrés : entre 2003 et 2010, la proportion d'anesthésistes prenant une garde exclusive en maternité est passée pour les maternités niveau 3 de 57 à $85 \%$, la présence d'une garde d'interne d'anesthésie de 27 à $52 \%$. L'utilisation de l'anesthésie locorégionale pour les accouchements a atteint $80 \%$ en 2010 tous établissements confondus.

L'anesthésie péridurale obstétricale assure le confort de la femme, mais également la sécurisation de l'accouchement. La diminution du recours à l'anesthésie générale en cours de travail a fait diminuer de manière drastique la mortalité maternelle d'origine anesthésique [2]. Cette sécurisation de l'accouchement est d'autant plus cruciale que la démographie des femmes a changé avec un vieillissement des patientes : augmentation de la moyenne d'âge à 29,9 ans et une augmentation de l'indice de masse corporelle : $10 \%$ des parturientes sont obèses et $17,3 \%$ en surpoids, toujours d'après le récent rapport périnatal 2010. La stabilité du taux de mortalité maternelle au cours des six dernières années traduit probablement une amélioration des prises en charge

E. Morau $(\bowtie)$

Département d'anesthésie réanimation $\mathrm{D}$,

secteur gynécologie et obstétrique, hôpital Arnaud-de-Villeneuve,

371, avenue du Doyen-Gaston-Giraud,

F-34295 Montpellier cedex 05, France

e-mail : e-morau@chu-montpellier.fr dans ce contexte. L'analgésie péridurale permet d'offrir également la possibilité d'accoucher par voie basse à des femmes pour lesquelles l'effort que représenterait un accouchement sans analgésie serait une contre-indication (patientes à comorbidités cardiaque, respiratoire, neurologique ou ophtalmologique). L'analgésie péridurale dans cette optique de sécurisation de l'accouchement doit donc pouvoir être proposée aux femmes d'autant que son retentissement sur le déroulement du travail est modeste, voire nul [3].

Les résultats favorables sur la prise en charge anesthésique en obstétrique sont donc la conjonction d'actions d'amélioration de l'organisation des soins et de dispositifs réglementaires : la rédaction de décrets de loi [4,5] a imposé aux maternités de s'équiper et de s'organiser pour permettre la sécurité maternelle lors de l'accouchement. Toutes les femmes enceintes bénéficient d'une consultation préanesthésique d'autant plus précoce qu'elles sont porteuses de pathologies à risque pour la grossesse ou l'accouchement. En termes d'organisation également, il a été montré que la présence permanente d'un anesthésiste-réanimateur au sein d'une maternité était un facteur indépendant positif sur la qualité de prise en charge d'une hémorragie du postpartum [6].

L'organisation des maternités en réseaux de santé a permis également un meilleur maillage entre les établissements et une orientation adéquate de l'enfant à naître vers un niveau de soins lui correspondant. Néanmoins, ce maillage centré sur l'enfant ne prend pas en compte les conditions d'accueil de la mère et c'est à chaque réseau de réfléchir à une cartographie de plateaux techniques optimaux pour l'accueil de mères à risque ou en cours de décompensation (accès à un bloc opératoire et à une réanimation adulte à proximité immédiate de la maternité, disponibilité d'un plateau technique d'embolisation, accès rapide aux produits sanguins labiles et au laboratoire de biologie).

Dans cette réflexion, les anesthésistes-réanimateurs sont également des interlocuteurs de choix de par leur compétence en réanimation. Néanmoins, l'implication des anesthésistes dans les réseaux périnataux de France, bien que non 
chiffrée, ne semble pas majeure (peut-être parce que $90 \%$ des transferts environ sont réalisés pour cause fœtale). Pourtant, ces réseaux formalisés permettent une meilleure communication entre les équipes d'une même région, favorisent la mise en œuvre de protocoles communs, la définition de critères de transfert et tous ces facteurs concourent à une meilleure organisation sécuritaire autour de la naissance.

Les anesthésistes œuvrant en obstétrique sont fédérés au plan national par le club d'anesthésie et réanimation obstétricale créé par Jeanne Seebacher dans les années 1970 pour promouvoir et partager les données naissantes de l'analgésie obstétricale. Ce « club » est désormais formalisé avec une organisation autour d'une présidence assurée successivement par des personnalités fortes de l'anesthésie obstétricale française, Maryse Palot, Dan Benhamou, Frédéric Mercier, Dominique Chassard, et deux rencontres nationales annuelles avec pour objectif de partager l'information scientifique, de réfléchir à l'évolution des pratiques de la spécialité dans un climat de convivialité.

L'évolution de l'anesthésie obstétricale se poursuit vers le développement des compétences en réanimation surspécialisée avec la création d'unités de soins continus obstétricaux au sein des maternités pour assurer une prise en charge optimale des femmes développant une complication pendant la grossesse ou l'accouchement (prééclampsie, hémorragie, etc.) ou décompensant des comorbidités préexistantes. Ces unités sont sous la coresponsabilité des obstétriciens et des anesthésistes mettant en évidence la complémentarité des deux spécialités, la nécessaire confiance réciproque, et la bonne communication au sein de l'équipe.

En quelques dizaines d'années, l'anesthésiste-réanimateur est devenu un interlocuteur de choix en salle de naissance.
Organisation et anticipation plaident pour sa présence permanente et son positionnement en plus du couple obstétricienpédiatre dans la prise en charge de la mère. Ses compétences en réanimation permettent de réfléchir à de nouveaux modes de prise en charge des patientes ayant des pathologies plus complexes permettant de les maintenir dans les maternités. L'implication au sein des réseaux périnataux reste un objectif à atteindre pour organiser cette nouvelle dynamique à l'échelle d'une région.

Remerciements chaleureux au Pr Dan Benhamou pour sa relecture attentive.

\section{Références}

1. Bondel B, Kermarrec M (2011) La situation périnatale en France. Études et résultats $\mathrm{n}^{\mathrm{o}} 775$

2. Maternal deaths from anaesthesia (2005) An extract from why mothers die 2000-2002, the confidential enquiries into maternal deaths in the United Kingdom. Br J Anaesth 94:417-424

3. Cambic C, Wong C (2010) Labour analgesia and obstetric outcomes. Br J Anaesth 105(suppl 1):i50-i60

4. Décret $\mathrm{n}^{\circ}$ 94-1050 du 5 décembre 1994, relatif aux conditions techniques de fonctionnement des établissements de santé en ce qui concerne la pratique de l'anesthésie et modifiant le code de la santé publique

5. Décret $\mathrm{n}^{\circ}$ 98-900 du 9 octobre 1998, relatif aux conditions techniques de fonctionnement auxquelles doivent satisfaire les établissements de santé pour être autorisés à pratiquer les activités d'obstétrique, de néonatologie ou de réanimation néonatale et modifiant le code de la santé publique

6. Bouvier-Colle MH, El Joud D, Varnoux N, Goffinet F (2001) Evaluation of the quality of care for severe obstetrical haemorrhage in three French regions. Br J Obstet Gynaecol 108:898-903 